\title{
Reply to: Are stressful childhood experiences relevant in non-monosexual women?
}

\author{
Tonje J. Persson ${ }^{1 *}$, James G. Pfaus ${ }^{1}$, and Andrew G. Ryder ${ }^{2,3 * *}$ \\ ${ }^{1}$ Center for Studies in Behavioral Neurobiology, Department of Psychology (SP 244), Concordia University, 7141 \\ Sherbrooke Street West, Montréal, QC H4B 1R6, Canada \\ ${ }^{2}$ Centre for Clinical Research in Health, Department of Psychology, Concordia University, 7141 Sherbrooke Street West, \\ Montréal, QC H4B 1R6, Canada \\ ${ }^{3}$ Culture and Mental Health Research Unit and Lady Davis Institute, Jewish General Hospital, Canada
}

We thank the commentator for his thoughtful response (Schneeberger, 2015) to our study entitled, "Explaining Mental Health Disparities for Non-monosexual Women: Abuse History and Risky Sex, or the Burdens of Nondisclosure?" (Persson et al., 2014) To summarize, Schneeberger (2015) highlights three aspects of our methodological approach: (1) how the participants were grouped; (2) how sexual orientation was evaluated; and (3) how a history of childhood abuse was assessed. We will reflect on these three issues while further considering future research directions in the study of female sexual orientation and childhood abuse.

We grouped our participants into one of two sexual orientation categories, namely, monosexual versus nonmonosexual. The commentator describes this novel methodology as "forward-looking" because "it focuses on the knowledge that analyzing LGBT populations as a whole neglects many noteworthy features that affect only subgroups." As has been demonstrated by research, non-monosexual women may report worse mental health, may experience more lifetime adversity, and may face more stigma and discrimination than monosexual women (Friedman et al., 2011, 2014; Kerr et al., 2013). Further, bisexual individuals may not feel part of the LGBT community (Herek et al., 2010). In short, although non-monosexual women have often been grouped into mono sexual categories, doing so may compromise ecological validity. Schneeberger (2015) argues that

DOI of original article: http://dx.doi.org/10.1016/j.socscimed. 2014.10.008.

* Corresponding author.

** Corresponding author. Centre for Clinical Research in Health, Department of Psychology, Concordia University, 7141 Sherbrooke Street West, Montréal, QC H4B 1R6, Canada.

E-mail addresses: tj_perss@hotmail.com (T.J. Persson), jim.pfaus@concordia.ca (J.G. Pfaus), andrew.ryder@concordia.ca (A.G. Ryder). future studies "should follow the lead" of our study by analyzing subgroups of sexual minority women. We agree, and would go further: future studies should continue to explore not only, how mostly lesbian, bisexual, and mostly heterosexual women might differ from monosexual women, but also how women in these more fine-grained categories might differ from one another. We recommend, if participant numbers allow, a five-category approach (heterosexual, mostly heterosexual, bisexual, mostly lesbian, lesbian) for sexual orienta tion self-identification (Vrangalova and Savin-Williams, 2012). In addition, sexual orientation research should move beyond the North American or Western European context. As we pointed out in the original paper, combining lesbian and heterosexual women may not be valid in societies less accepting of lesbian/gay rights than, for example, in Canada. In short, researchers need to account for the sociocultural context of study participants (Ryder et al., 2011).

Outcomes in sexual orientation research may vary depending on how sexual orientation is defined and assessed. For instance, McCabe et al. (2012) found that when using a three-category approach, namely heterosexual, bisexual, gay/lesbian, bisexual participants reported the highest level of substance abuse. How ever, when using a five-category approach, namely heterosexual, mostly heterosexual, bisexual, mostly lesbian, lesbian, the mostly heterosexual group was at the highest risk of substance abuse. Based on findings such as these, we opted to analyze results based on both sexual orientation self-identification and self-reported sexual behavior. Although the overall pattern of results did not differ based on which dimension of sexual orientation was used, we suggest that future studies continue to take this multi-tiered approach. As pointed out by Schneeberger (2015): “Using this comprehensive approach [... ] gives more accurate information about the actual sexual behaviors in the analyzed group and focuses more on the people who do not clearly identify themselves with a monosexual orientation group." 
PERSSON ET AL.

Future studies should continue to explore how mental health outcomes may vary by non-monosexual behavior versus by identifying as mostly heterosexual, bisexual, or mostly lesbian. For example, considering that "bisexual" appears to be a stigmatized label (Friedman et al., 2014; Zivony and Lobel, 2014), it is possible that those who behave bisexually without identifying as bisexual may be at lower risk of poor mental health compared to those who both behave bisexually and identify as bisexual.

Schneeberger (2015) raises the very important question of whether stressful childhood experiences are relevant in non monosexual women. He addresses how our findings, in regards to the association(s) between sexual orientation, childhood abuse, risky sexual behavior, and mental health, may be limited by the ways in which childhood abuse was defined and analyzed. We agree with him that future studies should assess traumatic chil hood experiences in a more comprehensive way than in our study. In addition, we concur that it may be important to define childhood abuse dimensionally, rather than taking the categorical approach used in our study. Schneeberger (2015) points out how a "cumulative approach" to defining traumatic childhood experiences among sexual minorities may be the most likely "to yield accurate results." A recent study investigating the association between discrimination and mental health among sexual minorities found that sexual orientation discrimination was only linked to higher odds of a past year mental health disorder in combination with other forms of discrimination (racial, ethnic, gender) (Bostwick et al., 2014). Findings such as these underline the importance of investigating parts of larger constructs, such as "sexual orienta tion," "childhood abuse," and "discrimination."

Research on sexual orientation minorities, generally, and research on non-monosexual women, specifically, may benefit from a methodological approach in which constructs such as "sexual orientation" and "childhood abuse" are studied in a more fine-grained way. In order to analyze these separate components, study designs have to allow for participants to define themselves along several dimensions, such as, identity and behavior. The results of these studies then need to be understood and interpreted in context-within the participants' local sociocultural worlds.

\section{Acknowledgments}

TJP was supported by a doctoral scholarship from the Canadian Institutes of Health Research.

\section{References}

Bostwick, W.B., Boyd, C.J., Hughes, T.L., West, B.T., McCabe, S.E., 2014. Discrimination and mental health among lesbian, gay, and bisexual adults in the United States. Am. J. Orthopsychiatry 84(1), 35-45.

Friedman, M.S., Marshal, M.P., Guadamuz, T.E., Wei, C., Wong, C.F., Saewyc, E.M., Stall, R., 2011. A meta-analysis of disparities in childhood sexual abuse, parental physical abuse, and peer victimization among sexual minority and sexual nonminority individuals. Am. J. Public Health 101(8), 1481-1494. http:// dx.doi.org/10.2105/ajph.2009.190009.

Friedman, M.R., Dodge, B., Schick, V., Herbenick, D., Hubach, R.D., Bowling, J., Reece, M., 2014. From bias to bisexual health disparities: attitudes toward bisexual men and women in the United States. LGBT Health 2, 1-10. http://dx.doi.org/10.1089/lgbt.2014.0005.

Herek, G.M., Norton, A.T., Allen, T.J., Sims, C.L., 2010. Demographic, psychological, and social characteristics of self-identified lesbian, gay, and bisexual adults in a US probability sample. Sex. Res. Soc. Policy: A J. NSRC 7(3), 176-200.

Kerr, D.L., Santurri, L., Peters, P., 2013. A comparison of lesbian, bisexual, and het erosexual college undergraduate women on selected mental health issues. J. Am. Coll. Health 61(4), 185-194. http://dx.doi.org/10.1080/ 07448481.2013 .787619 .

McCabe, S.E., Hughes, T.L., Bostwick, W., Morales, M., Boyd, C.J., 2012. Measurement of sexual identity in surveys: implications for substance abuse research. Arch. Sex. Behav. 41(3), 649-657. http://dx.doi.org/10.1007/s10508011-9768-7.

Persson, T.J., Pfaus, J.G., Ryder, A.G., 2015. Explaining mental health disparities for non-monosexual women: abuse history and risky sex, or the burdens of nondisclosure? Soc. Sci. Med. 128, 366-373.

Ryder, A.G., Ban, L.M., Chentsova-Dutton, Y.E., 2011. Towards a cultural-clinical psychology. Soc. Personality Psychol. Compass 5(12), 960-975.

Schneeberger, A.R., 2015. Are stressful childhood experiences relevant in non monosexual women? A comment on Persson, T.J., Pfaus, J.G., \& Ryder, A.G. (2014). Soc. Sci. Med. 128, 334-335.

Vrangalova, Z., Savin-Williams, R.C., 2012. Mostly heterosexual and mostly gay/ lesbian: evidence for new sexual orientation identities. Arch. Sex. Behav. 41, 85-101.

Zivony, A., Lobel, T., 2014. The invisible stereotypes of bisexual men. Arch. Sex. Behav. 1-12. http://dx.doi.org/10.1007/s10508-014-0263-9. 